\title{
Precision Measurement of Cosmic-Ray Nitrogen and its Primary and Secondary Components with the Alpha Magnetic Spectrometer on the International Space Station
}

M. Aguilar,${ }^{27}$ L. Ali Cavasonza, ${ }^{1}$ B. Alpat,${ }^{32}$ G. Ambrosi,${ }^{32}$ L. Arruda,${ }^{25}$ N. Attig, ${ }^{22}$ S. Aupetit,,${ }^{17}$ P. Azzarello, ${ }^{16}$ A. Bachlechner, ${ }^{1}$ F. Barao, ${ }^{25}$ A. Barrau, ${ }^{17}$ L. Barrin, ${ }^{15}$ A. Bartoloni, ${ }^{37}$ L. Basara, ${ }^{35}$ S. Başeğmez-du Pree,,${ }^{6,2}$ M. Battarbee, ${ }^{44}$ R. Battiston, ${ }^{35,36, a}$ U. Becker, ${ }^{10}$ M. Behlmann, ${ }^{10}$ B. Beischer, ${ }^{1}$ J. Berdugo, ${ }^{27}$ B. Bertucci,,${ }^{32,33}$ K. F. Bindel,${ }^{23}$ V. Bindi, ${ }^{20}$ W. de Boer, ${ }^{23}$ K. Bollweg, ${ }^{21}$ V. Bonnivard, ${ }^{17}$ B. Borgia, ${ }^{37,38}$ M. J. Boschini, ${ }^{29}$ M. Bourquin, ${ }^{16}$ E. F. Bueno, ${ }^{39}$ J. Burger, ${ }^{10}$ W. J. Burger, ${ }^{35}$ X. D. Cai,,${ }^{10}$ M. Capell, ${ }^{10}$ S. Caroff, ${ }^{3}$ J. Casaus, ${ }^{27}$ G. Castellini, ${ }^{14}$ F. Cervelli, ${ }^{34}$ Y. H. Chang, ${ }_{11}$ A. I. Chen, ${ }^{10}$ G. M. Chen, ${ }^{6}$ H. S. Chen, ${ }^{6,7}$ Y. Chen, ${ }^{16}$ L. Cheng,${ }^{40}$ H. Y. Chou, ${ }^{11}$ E. Choumilov, ${ }^{10}$ V. Choutko, ${ }^{10}$ C. H. Chung,,${ }^{1}$ C. Clark, ${ }^{21}$ R. Clavero, ${ }^{24}$ G. Coignet, ${ }^{3}$ C. Consolandi,${ }^{20}$ A. Contin,${ }^{8,9}$ C. Corti, ${ }^{20}$ W. Creus, ${ }^{43}$ M. Crispoltoni, ${ }^{32,33}$ Z. Cui, ${ }^{40}$ K. Dadzie, ${ }^{10}$ Y. M. Dai, ${ }^{5}$ A. Datta, ${ }^{20}$ C. Delgado, ${ }^{27}$ S. Della Torre, ${ }^{29}$ M. B. Demirköz,${ }^{2}$ L. Derome, ${ }^{17}$ S. Di Falco, ${ }^{34}$ F. Dimiccoli, ${ }^{35,15}$ C. Díaz, ${ }^{27}$ P. von Doetinchem,${ }^{20}$ F. Dong, ${ }^{31}$ F. Donnini, ${ }^{32, b}$ M. Duranti, ${ }^{32}$ A. Egorov, ${ }^{10}$ A. Eline, ${ }^{10}$ T. Eronen, ${ }^{44}$ J. Feng, ${ }^{10}$ E. Fiandrini, ${ }^{32,33}$ P. Fisher, ${ }^{10}$ V. Formato, ${ }^{32}$ Y. Galaktionov,${ }^{10}$ G. Gallucci, ${ }^{34}$ R. J. García-López, ${ }^{24}$ C. Gargiulo, ${ }^{15}$ H. Gast,${ }^{1}$ I. Gebauer, ${ }^{23}$ M. Gervasi, ${ }^{29,30}$ A. Ghelfi, ${ }^{17}$ F. Giovacchini ${ }^{27}$ D. M. Gómez-Coral, ${ }^{28}$ J. Gong, ${ }^{31}$ C. Goy, ${ }^{3}$ V. Grabski, ${ }^{28}$ D. Grandi, ${ }^{29,30}$ M. Graziani, ${ }^{23}$ K. H. Guo, ${ }^{19}$ S. Haino, ${ }^{43}$ K. C. Han,${ }^{26}$ Z. H. He ${ }^{19}$ M. Heil,${ }^{10}$ T. H. Hsieh, ${ }^{10}$ H. Huang, ${ }^{43, c}$ Z. C. Huang, ${ }^{19}$ M. Incagli, ${ }^{34}$ Yi Jia, ${ }^{10}$ H. Jinchi, ${ }^{26}$ K. Kanishev ${ }^{35,15}$ B. Khiali, ${ }^{11,32, \mathrm{~b}}$ Th. Kirn, ${ }^{1}$ C. Konak, ${ }^{2}$ O. Kounina, ${ }^{10}$ A. Kounine, ${ }^{10}$ V. Koutsenko, ${ }^{10}$ A. Kulemzin, ${ }^{10}$ G. La Vacca, ${ }^{29,30}$ E. Laudi, ${ }^{15}$ G. Laurenti, ${ }^{8}$ I. Lazzizzera, ${ }^{35,36}$ A. Lebedev, ${ }^{10}$ H. T. Lee, ${ }^{42}$ S. C. Lee,${ }^{43}$ C. Leluc, ${ }^{16}$ H. S. Li, ${ }^{41}$ J. Q. Li ${ }^{31}$ Q. Li ${ }^{31}$ T. X. Li ${ }^{19}$ Z. H. Li ${ }^{6}{ }^{6}$ Z. Y. Li, ${ }^{43, d}$ C. H. Lin, ${ }^{43}$ P. Lipari, ${ }^{37}$ T. Lippert, ${ }^{22}$ D. Liu, ${ }^{11}$ Hu Liu, ${ }^{10}$ Z. Liu, ${ }^{16}$ V. D. Lordello, ${ }^{39}$ S. Q. Lu, ${ }^{43, d}$ Y. S. Lu, ${ }^{6}$ K. Luebelsmeyer, ${ }^{1}$ F. Luo, ${ }^{40}$ J. Z. Luo, ${ }^{31}$ S. S. Lyu, ${ }^{19}$ F. Machate, ${ }^{1}$ C. Mañá, ${ }^{27}$ J. Marín, ${ }^{27}$ T. Martin, ${ }^{21}$ G. Martínez, ${ }^{27}$ N. Masi, ${ }^{8}$ D. Maurin, ${ }^{17}$ A. Menchaca-Rocha, ${ }^{28}$ Q. Meng, ${ }^{31}$ V. M. Mikuni, ${ }^{39}$ D. C. Mo, ${ }^{19}$ P. Mott, ${ }^{21}$ L. Mussolin, ${ }^{32,33}$ T. Nelson, ${ }^{20}$ J. Q. Ni, ${ }^{19}$ N. Nikonov, ${ }^{1}$ F. Nozzoli, ${ }^{35}$ A. Oliva, ${ }^{27}$ M. Orcinha, ${ }^{25}$ M. Palermo, ${ }^{20}$ F. Palmonari, ${ }^{8,9}$ C. Palomares, ${ }^{27}$ M. Paniccia, ${ }^{16}$ M. Pauluzzi, ${ }^{32,33}$ S. Pensotti, ${ }^{29,30}$ C. Perrina, ${ }^{16}$ H. D. Phan, ${ }^{10}$ N. Picot-Clemente, ${ }^{13}$ F. Pilo,${ }^{34}$ V. Plyaskin, ${ }^{10}$ M. Pohl,${ }^{16}$ V. Poireau, ${ }^{3}$ L. Quadrani,${ }^{8,9}$ X. M. Qi,${ }^{19}$ X. Qin,${ }^{10}$ Z. Y. Qu, ${ }^{43, \mathrm{e}}$ T. Räihä, ${ }^{1}$ P. G. Rancoita, ${ }^{29}$ D. Rapin, ${ }^{16}$ J. S. Ricol,${ }^{17}$ S. Rosier-Lees, ${ }^{3}$ A. Rozhkov, ${ }^{10}$ D. Rozza, ${ }^{29,30}$ R. Sagdeev, ${ }^{12}$ S. Schael, ${ }^{1}$ S. M. Schmidt, ${ }^{22}$ A. Schulz von Dratzig, ${ }^{1}$ G. Schwering, ${ }^{1}$ E. S. Seo, ${ }^{13}$ B. S. Shan, ${ }^{4}$ J. Y. Shi, ${ }^{31}$ T. Siedenburg, ${ }^{1}$ J. W. Song, ${ }^{40}$ M. Tacconi, ${ }^{29,30}$ X. W. Tang, ${ }^{6}$ Z. C. Tang, ${ }^{6}$ D. Tescaro, ${ }^{24}$ J. Tian, ${ }^{32,33}$ Samuel C. C. Ting, ${ }^{10,15}$ S. M. Ting, ${ }^{10}$ N. Tomassetti, ${ }^{32,33}$ J. Torsti, ${ }^{44}$ T. Urban, ${ }^{21}$ V. Vagelli, ${ }^{32,33}$ E. Valente, ${ }^{37,38}$ E. Valtonen, ${ }^{44}$ M. Vázquez Acosta, ${ }^{24}$ M. Vecchi, ${ }^{39,18}$ M. Velasco, ${ }^{27}$ J. P. Vialle, ${ }^{3}$ L. Q. Wang, ${ }^{40}$ N. H. Wang, ${ }^{40}$ Q. L. Wang, ${ }^{5}$ X. Wang, ${ }^{10}$ X. Q. Wang, ${ }^{6,7}$ Z.X. Wang, ${ }^{19}$ C. C. Wei,${ }^{43, f}$ J. Wei,${ }^{16}$ Z. L. Weng, ${ }^{10}$ K. Whitman, ${ }^{20}$ H. Wu, ${ }^{31}$ R. Q. Xiong, ${ }^{31}$ W. Xu, ${ }^{10}$ Q. Yan, ${ }^{10}$ M. Yang, ${ }^{6}$ Y. Yang, ${ }^{41}$ H. Yi, ${ }^{31}$ Y. J. Yu, ${ }^{5}$ Z. Q. Yu, ${ }^{6}$ M. Zannoni, ${ }^{29,30}$ S. Zeissler, ${ }^{23}$ C. Zhang, ${ }^{6}$ F. Zhang, ${ }^{6}$ J. Zhang, ${ }^{10, c}$ J. H. Zhang, ${ }^{31}$ S. W. Zhang, ${ }^{6,7}$ Z. Zhang, ${ }^{10}$ Z. M. Zheng, ${ }^{4}$ H. L. Zhuang, ${ }^{6}$ V. Zhukov, ${ }^{1}$ A. Zichichi, ${ }^{8,9}$ N. Zimmermann, ${ }^{1}$ and P. Zuccon ${ }^{10,35,36}$

(AMS Collaboration)

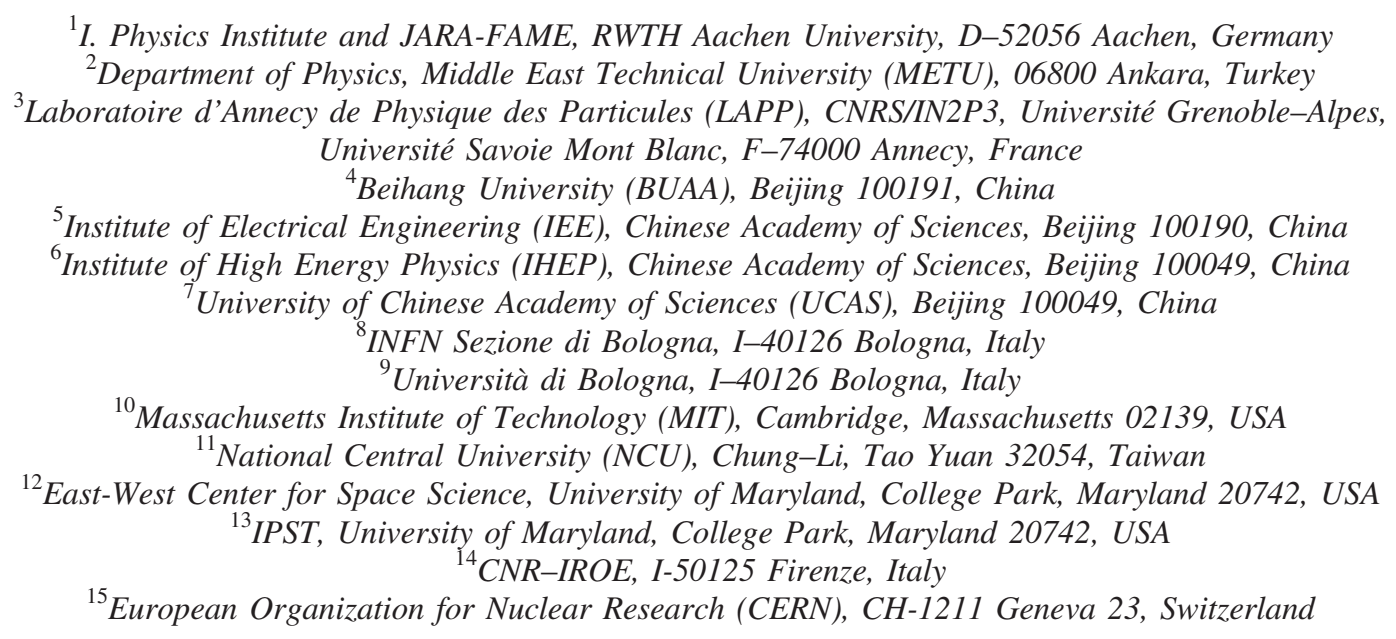

0031-9007/18/121(5)/051103(8)

$051103-1$

Published by the American Physical Society 




(Received 16 April 2018; revised manuscript received 6 June 2018; published 31 July 2018)

A precision measurement of the nitrogen flux with rigidity (momentum per unit charge) from $2.2 \mathrm{GV}$ to $3.3 \mathrm{TV}$ based on $2.2 \times 10^{6}$ events is presented. The detailed rigidity dependence of the nitrogen flux spectral index is presented for the first time. The spectral index rapidly hardens at high rigidities and becomes identical to the spectral indices of primary He, C, and $\mathrm{O}$ cosmic rays above $\sim 700 \mathrm{GV}$. We observed that the nitrogen flux $\Phi_{\mathrm{N}}$ can be presented as the sum of its primary component $\Phi_{\mathrm{N}}^{P}$ and secondary component $\Phi_{\mathrm{N}}^{S}, \Phi_{\mathrm{N}}=\Phi_{\mathrm{N}}^{P}+\Phi_{\mathrm{N}}^{S}$, and we found $\Phi_{\mathrm{N}}$ is well described by the weighted sum of the oxygen flux $\Phi_{\mathrm{O}}$ (primary cosmic rays) and the boron flux $\Phi_{\mathrm{B}}$ (secondary cosmic rays), with $\Phi_{\mathrm{N}}^{P}=$ $(0.090 \pm 0.002) \times \Phi_{\mathrm{O}}$ and $\Phi_{\mathrm{N}}^{S}=(0.62 \pm 0.02) \times \Phi_{\mathrm{B}}$ over the entire rigidity range. This corresponds to a change of the contribution of the secondary cosmic ray component in the nitrogen flux from $70 \%$ at a few $\mathrm{GV}$ to $<30 \%$ above $1 \mathrm{TV}$.

DOI: 10.1103/PhysRevLett.121.051103

Nitrogen nuclei in cosmic rays are thought to be produced both in astrophysical sources, mostly via the CNO cycle [1,2], and by the collisions of heavier nuclei

Published by the American Physical Society under the terms of the Creative Commons Attribution 4.0 International license. Further distribution of this work must maintain attribution to the author(s) and the published article's title, journal citation, and DOI. with the interstellar medium [3]. Therefore the nitrogen flux $\Phi_{\mathrm{N}}$ is expected to contain both primary $\Phi_{\mathrm{N}}^{P}$ and secondary $\Phi_{\mathrm{N}}^{S}$ components. Precise knowledge of the primary component of cosmic nitrogen provides important insights into the details of nitrogen production in astrophysical sources, while precise knowledge of the secondary component of the cosmic nitrogen provides insights into the details of propagation processes of cosmic rays in the Galaxy. Over the last 50 years, few experiments have measured the nitrogen flux [4-7]. 
Typically, these measurements have errors larger than $40 \%-50 \%$ above $100 \mathrm{GV}$.

Precision measurements of the primary $\mathrm{He}, \mathrm{C}$, and $\mathrm{O}$ cosmic ray fluxes and of the secondary $\mathrm{Li}, \mathrm{Be}$, and $\mathrm{B}$ cosmic ray fluxes by the Alpha Magnetic Spectrometer (AMS) have been reported [8,9] with typical errors of $2 \%-4 \%$ at $100 \mathrm{GV}$.

To determine the primary and secondary components in the nitrogen flux, we have chosen the rigidity dependence of the oxygen flux as characteristic of primary fluxes and the rigidity dependence of the boron flux as characteristic of secondary fluxes. The secondary component of the oxygen flux is the lowest (a few percent $[10,11]$ ) among $\mathrm{He}, \mathrm{C}$, and $\mathrm{O}$. The boron flux has no primary contribution and is mostly produced from the interactions of primary cosmic rays $\mathrm{C}$ and $\mathrm{O}$ with interstellar matter.

In this Letter we report the precision measurement of the nitrogen flux in cosmic rays in the rigidity range from $2.2 \mathrm{GV}$ to $3.3 \mathrm{TV}$ based on data collected by the AMS during the first five years (May 19, 2011 to May 26, 2016) of operation aboard the International Space Station (ISS). The total flux error is $4 \%$ at $100 \mathrm{GV}$.

Detector.-The layout and description of the AMS detector are presented in Ref. [12]. The key elements used in this measurement are the permanent magnet [13], the silicon tracker [14], and the four planes of time of flight (TOF) scintillation counters [15]. Further information on the layout and the performance of the silicon tracker and the TOF is included in Refs. [16,17]. AMS also contains a transition radiation detector (TRD), a ring imaging Čerenkov detector, an electromagnetic calorimeter, and an array of 16 anticoincidence counters.

Nitrogen nuclei traversing AMS were triggered as described in Ref. [18]. The trigger efficiency has been measured to be $>98 \%$ over the entire rigidity range.

Monte Carlo (MC) simulated events were produced using a dedicated program developed by the collaboration based on the GEANT-4.10.1 package [19]. The program simulates electromagnetic and hadronic interactions of particles in the material of AMS and generates detector responses. The Glauber-Gribov model [19], tuned to reproduce the AMS helium data, see supplemental figures SM 1(a),1(b) in Ref. [18], was used for the description of the nuclei inelastic cross sections.

Event selection.-In the first five years AMS has collected $8.5 \times 10^{10}$ cosmic ray events. The collection time used in this analysis includes only those seconds during which the detector was in normal operating conditions and, in addition, AMS was pointing within $40^{\circ}$ of the local zenith and the ISS was outside of the South Atlantic Anomaly. Because of the geomagnetic field, this collection time increases with rigidity, becoming constant at $1.23 \times 10^{8}$ seconds above $30 \mathrm{GV}$.

Nitrogen events are required to be downward-going and to have a reconstructed track in the inner tracker which passes through $L 1$. In the highest rigidity region,
$R \geq 1.3 \mathrm{TV}$, the track is also required to pass through $L 9$. Track fitting quality criteria such as a $\chi^{2} /$ d.o.f. $<10$ in the bending coordinate are applied, similar to Refs. [18,20,21].

The measured rigidity is required to be greater than a factor of 1.2 times the maximum geomagnetic cutoff within the AMS field of view. The cutoff was calculated by backtracing [22] particles from the top of AMS out to 50 Earth's radii using the most recent IGRF model [23].

Charge measurements on $L 1$, the upper TOF, the inner tracker, the lower TOF, and, for $R>1.3 \mathrm{TV}, L 9$ are all required to be compatible with charge $Z=7$ as shown in Fig. 1 of the Supplemental Material [16] for the inner tracker and in Fig. 2 of the Supplemental Material [16] for the upper TOF for different rigidity ranges. With this selection, the charge confusion from noninteracting nuclei
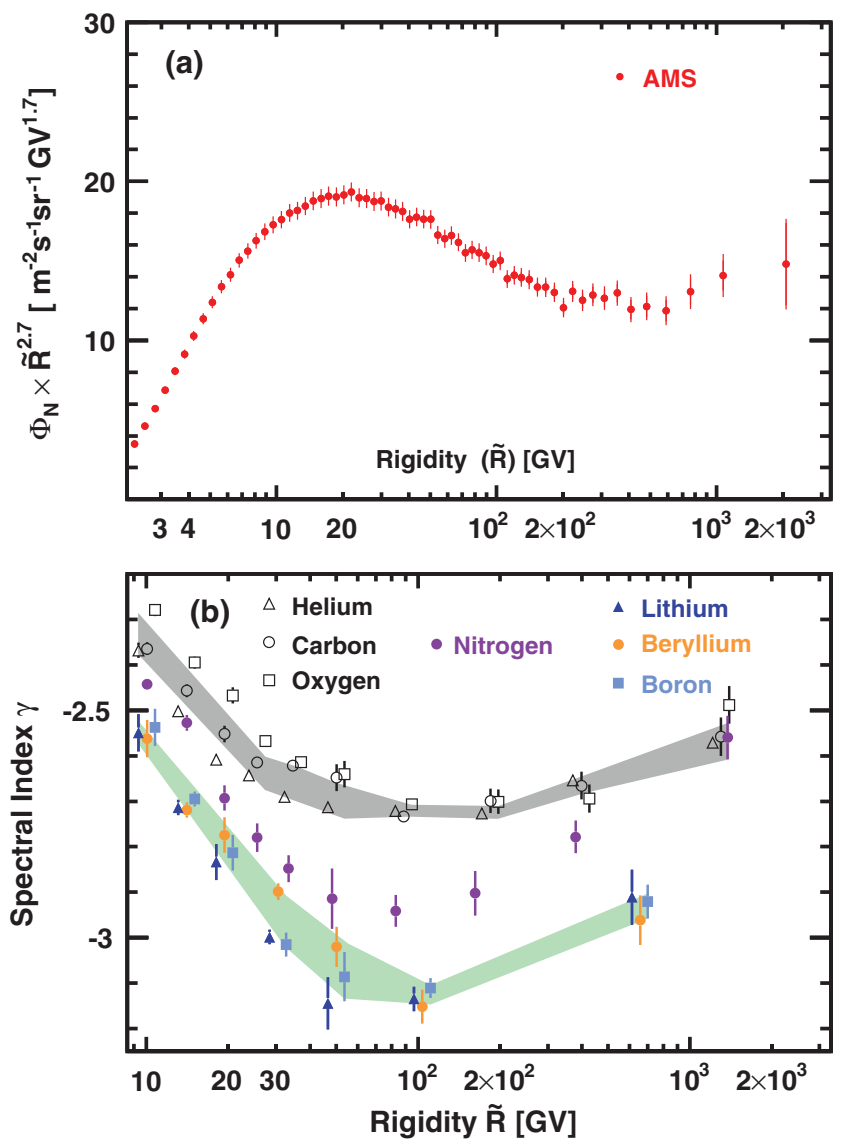

FIG. 1. (a) The AMS nitrogen flux $\Phi_{\mathrm{N}}[16]$ multiplied by $\tilde{R}^{2.7}$ with total errors as a function of rigidity. (b) The dependence of the nitrogen spectral index on rigidity together with the rigidity dependence of the spectral indices of primary $\mathrm{He}, \mathrm{C}$, and $\mathrm{O}$ cosmic rays and secondary $\mathrm{Li}, \mathrm{Be}$, and $\mathrm{B}$ cosmic rays. For clarity, the horizontal positions of the $\mathrm{Li}$ and $\mathrm{B}$ data points and $\mathrm{He}$ and $\mathrm{O}$ data points are displaced with respect to the $\mathrm{Be}$ and $\mathrm{C}$ data points, respectively. The shaded regions are to guide the eye. As seen, the nitrogen spectral index is situated between the primary and secondary cosmic ray spectral indices, hardens rapidly with rigidity above $\sim 100 \mathrm{GV}$ and becomes identical to the spectral indices of the primary cosmic rays above $\sim 700 \mathrm{GV}$. 


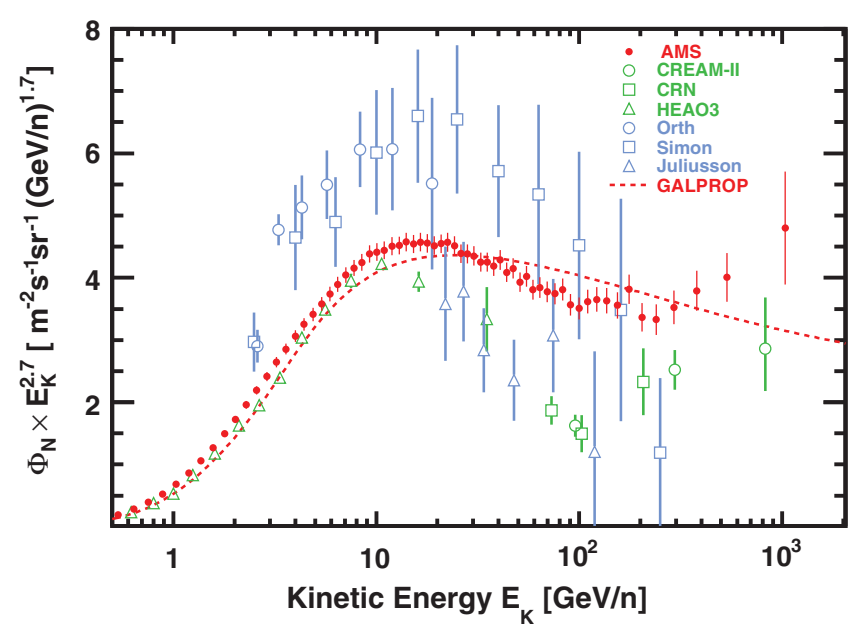

FIG. 2. The AMS nitrogen flux $\Phi_{\mathrm{N}}$ as a function of kinetic energy per nucleon $E_{K}$ multiplied by $E_{K}^{2.7}$ together with earlier measurements [4-7] and with the predictions of cosmic ray propagation model GALPROP [10] (dashed red curve).

is negligible $(<0.1 \%)$ over the whole rigidity range. This selection yields overall purities of $90 \%-95 \%$ depending on rigidity for nitrogen nuclei. The impurities have two sources. First, a residual background to nitrogen events results from the interactions of heavy nuclei such as $\mathrm{O}, \mathrm{F}$, and $\mathrm{Ne}$ in the material between $L 1$ and $L 2$ (the TRD and upper TOF). It has been evaluated by fitting the charge distribution from $L 1$ of events selected as nitrogen by the inner tracker with charge distribution templates of N, O, F, and $\mathrm{Ne}$. Then cuts are applied on the $L 1$ charge as shown in Fig. 3 the Supplemental Material [16]. The charge distribution templates are obtained using $L 2$. These templates contain only noninteracting events by requiring that $L 1$ and L3-L8 measure the same charge value. This residual background is $<5 \%$ over the entire rigidity range. Second, the background from $\mathrm{O}, \mathrm{F}$, and $\mathrm{Ne}$ interacting in materials above $L 1$ (thin support structures made of carbon fiber and aluminum honeycomb) has been estimated from simulation using MC samples generated according to AMS flux measurements [8,16,24]. This background is $<3 \%$ below $200 \mathrm{GV}$ and increases up to $6 \%$ at $3.3 \mathrm{TV}$. The simulation of nuclear interactions has been validated using data as shown in Fig. 4 of the Supplemental Material [16].

The overall uncertainty due to the total background subtraction is $<1.5 \%$ over the entire rigidity range. After background subtraction, we obtain $2.2 \times 10^{6}$ nitrogen nuclei.

Data analysis. - The isotropic flux $\Phi_{i}$ in the $i$ th rigidity bin $\left(R_{i}, R_{i}+\Delta R_{i}\right)$ is given by

$$
\Phi_{i}=\frac{N_{i}}{A_{i} \epsilon_{i} T_{i} \Delta R_{i}},
$$

where $N_{i}$ is the number of events corrected for bin-to-bin migration, $A_{i}$ is the effective acceptance, $\epsilon_{i}$ is the trigger
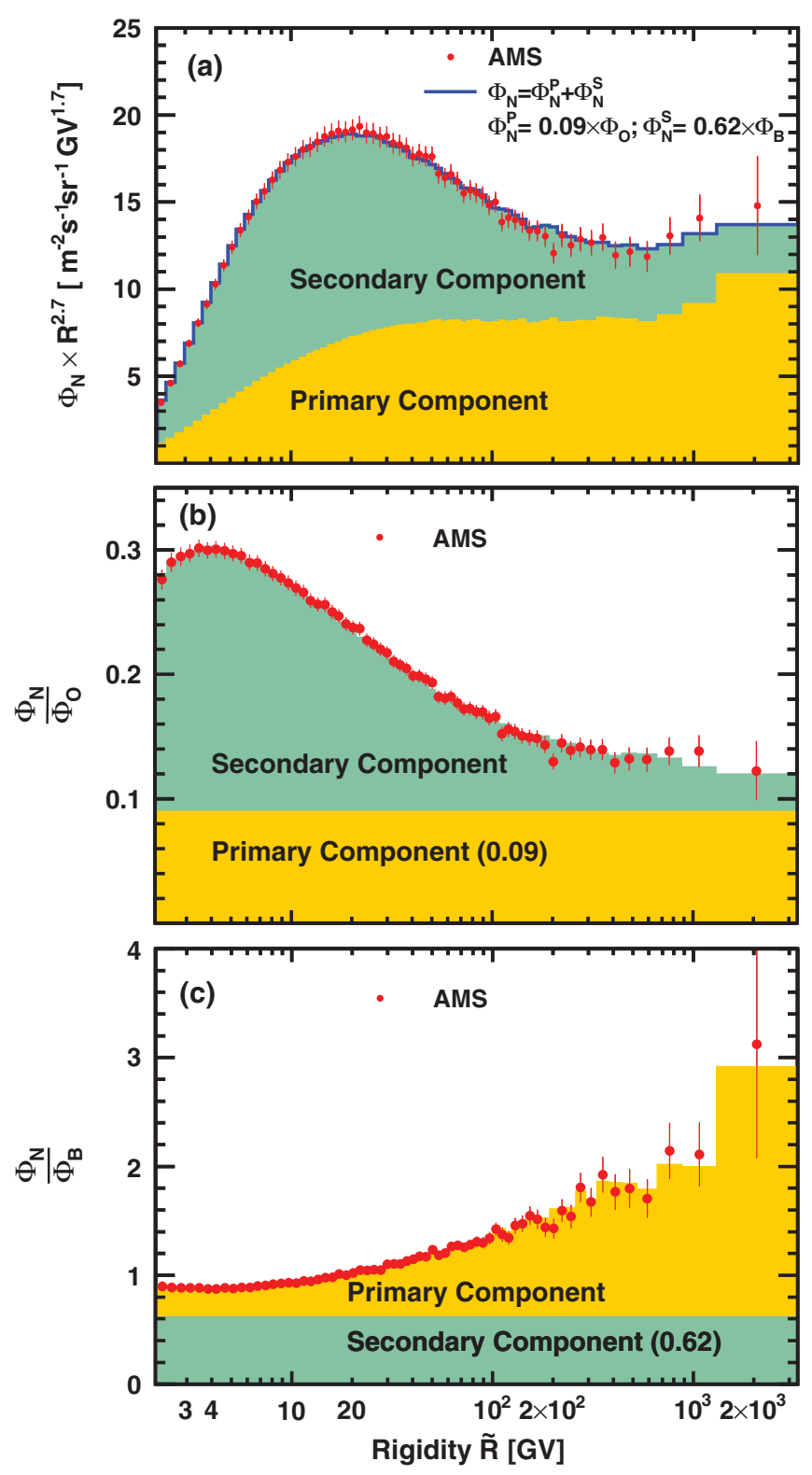

FIG. 3. (a) The AMS nitrogen flux $\Phi_{\mathrm{N}}$ fit to the weighted sum of the oxygen flux $\Phi_{\mathrm{O}}$ and the boron flux $\Phi_{\mathrm{B}}$ over the entire rigidity range. (b) The AMS $\left(\Phi_{\mathrm{N}} / \Phi_{\mathrm{O}}\right)$ ratio as a function of rigidity. (c) The AMS $\left(\Phi_{\mathrm{N}} / \Phi_{\mathrm{B}}\right)$ ratio as a function of rigidity. The contributions of the primary and secondary components are indicated by the shading (yellow and green, respectively). As seen from (b) and (c), the contribution of the secondary component in the nitrogen flux decreases, and the contribution of the primary component correspondingly increases, with rigidity.

efficiency, and $T_{i}$ is the collection time. In this Letter the nitrogen flux was measured in 66 bins from $2.2 \mathrm{GV}$ to 3.3 TV with bin widths chosen according to the rigidity resolution. Except for the first bin, the bin widths are identical with the bins used in the AMS publication on secondary cosmic rays [9].

The bin-to-bin migration of events was corrected using the unfolding procedure described in Ref. [20]. These 


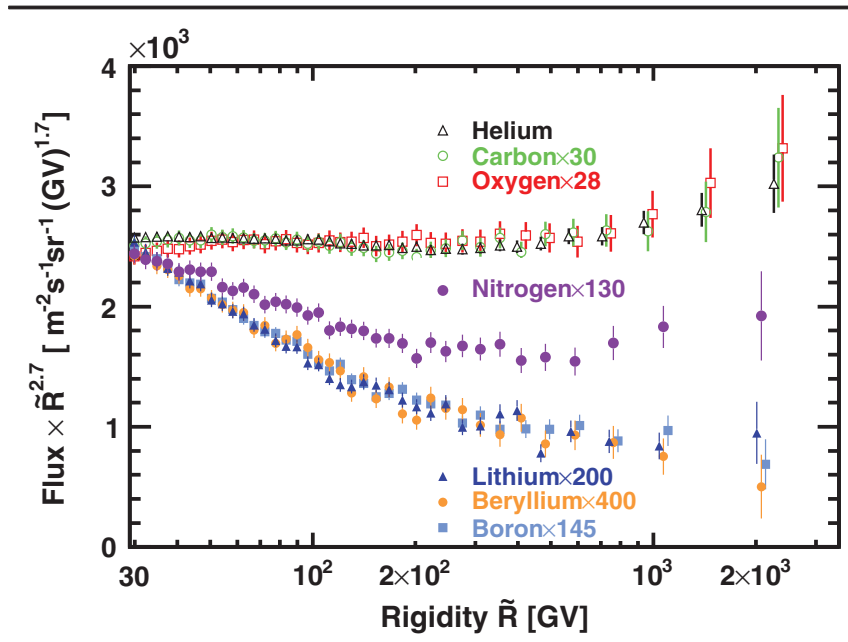

FIG. 4. Comparison of the AMS measurements of the primary cosmic ray fluxes [8] and the secondary cosmic rays fluxes [9] with the nitrogen flux [16] multiplied by $\tilde{R}^{2.7}$ with their total errors as functions of rigidity above $30 \mathrm{GV}$. For display purposes only, the $\mathrm{C}, \mathrm{O}, \mathrm{Li}, \mathrm{Be}, \mathrm{B}$, and $\mathrm{N}$ fluxes were rescaled as indicated. For clarity, the horizontal positions of the $\mathrm{He}, \mathrm{O}, \mathrm{Li}$, and $\mathrm{B}$ data points above $400 \mathrm{GV}$ are displaced. As seen, the three secondary fluxes have identical rigidity dependence above $30 \mathrm{GV}$ as do the three primary fluxes above $60 \mathrm{GV}$, but they are different from each other. The rigidity dependence of the nitrogen flux is distinctly different from the dependence of both the primary fluxes and the dependence of the secondary fluxes.

corrections, $\left(N_{i}-\boldsymbol{\aleph}_{i}\right) / \boldsymbol{\aleph}_{i}$, where $\boldsymbol{\aleph}_{i}$ is the number of observed events in bin $i$, are $+15 \%$ at $3 \mathrm{GV},+7 \%$ at $5 \mathrm{GV},-5 \%$ at $200 \mathrm{GV}$, and $-6 \%$ at $3.3 \mathrm{TV}$.

Extensive studies were made of the systematic errors. These errors include the uncertainties in the background estimations discussed above, the trigger efficiency, the geomagnetic cutoff factor, the acceptance calculation, the rigidity resolution function, and the absolute rigidity scale. The systematic error on the flux associated with the trigger efficiency measurement is $<0.7 \%$ over the entire rigidity range. The geomagnetic cutoff factor was varied from 1.0 to 1.4 , resulting in a negligible systematic uncertainty $(<0.1 \%)$ in the rigidity range below $30 \mathrm{GV}$.

The effective acceptances $A_{i}$ were calculated using MC simulation and corrected for small differences between the data and simulated events related to (a) event reconstruction and selection, namely, in the efficiency of velocity determination, track finding, charge determination, and tracker quality cuts and (b) the details of inelastic interactions of nitrogen nuclei in the AMS materials. The total corrections to the effective acceptance from the differences between the data and the MC simulation were found to be $<3 \%$, up to $500 \mathrm{GV}$ and $<5 \%$ at $3.3 \mathrm{TV}$. The systematic error on the flux associated with the reconstruction and selection is $<1 \%$ over the entire rigidity range. The material traversed by nuclei between $L 1$ and $L 9$ is composed primarily of carbon and aluminum [18]. The systematic error on the nitrogen flux due to uncertainties of inelastic cross sections for $\mathrm{N}+\mathrm{C}$ and $\mathrm{N}+\mathrm{O}$ was evaluated in a similar way as in Ref. [8] and discussed in detail in the data analysis section of the Supplemental Material of Ref. [16]. It was found to be $<3 \%$ up to $100 \mathrm{GV}$ and $4 \%$ at $3 \mathrm{TV}$.

The rigidity resolution function $\Delta(1 / R)$ for nitrogen has a pronounced Gaussian core characterized by width $\sigma$ and non-Gaussian tails more than $2.5 \sigma$ away from the center [18]. The resolution function has been verified with the procedures described in detail in Ref. [21]. As an example, Fig. 6 of the Supplemental Material of Ref. [16] shows that the measured tracker bending coordinate resolution of $5.5 \mu \mathrm{m}$ is in good agreement with the simulation. This yields the MDR of $3.5 \mathrm{TV}$ with $5 \%$ uncertainty and provides the uncertainties of $10 \%$ on the amplitudes of the non-Gaussian tails. The systematic error on the flux due to the rigidity resolution function was obtained by repeating the unfolding procedure while varying the width of the Gaussian core of the resolution function by $5 \%$ and by independently varying the amplitudes of the non-Gaussian tails by $10 \%$. The resulting systematic error on the flux is less than $1 \%$ below $150 \mathrm{GV}$ and $3 \%$ at $3.3 \mathrm{TV}$.

There are two contributions to the systematic uncertainty on the rigidity scale discussed in detail in Refs. $[8,20]$. The first is due to residual tracker misalignment. The second contribution arises from the magnetic field map measurement and magnetic field temperature corrections. The error on the flux due to this uncertainty is $<0.6 \%$ up to $100 \mathrm{GV}$ and 5\% in the last bin, 1.3-3.3 TV.

Much effort has been spent in understanding the systematic errors [18,20,21]. As an example, Fig. 7 of the Supplemental Material [16] shows the ratio of two measurements of the nitrogen flux from $2.2 \mathrm{GV}$ to $1.3 \mathrm{TV}$, one performed using events passing through $L 1$ to $L 8$ and the other using events passing through $L 1$ to $L 9$. The good agreement between the measurements verifies the systematic errors on unfolding, due to the difference in the resolution functions, and the systematic errors on acceptance, due to the difference in geometric factor and the amount of material traversed.

Most importantly, several independent analyses were performed on the same data sample by different study groups. The results of those analyses are consistent with this Letter.

Results.-The measured nitrogen flux including statistical and systematic errors is reported in Table I of the Supplemental Material [16] as a function of the rigidity at the top of the AMS detector. Figure 1(a) shows the nitrogen flux as a function of rigidity with the total errors, the quadratic sum of statistical and systematic errors. In this and the subsequent figures, the points are placed along the abscissa at $\tilde{R}$ calculated for a flux $\propto R^{-2.7}$ [25].

To examine the rigidity dependence of the flux, the detailed variation of the flux spectral index with rigidity was calculated in a model independent way from

$$
\gamma=d[\log (\Phi)] / d[\log (R)],
$$


over nonoverlapping rigidity intervals above $8.48 \mathrm{GV}$ with a variable width to have sufficient sensitivity to determine $\gamma$. The results are presented in Fig. 1(b) together with the spectral indices of primary cosmic rays $\mathrm{He}, \mathrm{C}$, and $\mathrm{O}$ [8] and of secondary cosmic rays $\mathrm{Li}, \mathrm{Be}$, and $\mathrm{B}$ [9]. As seen, the nitrogen spectral index is situated between the primary and secondary cosmic ray spectral indices, hardens rapidly with rigidity above $\sim 100 \mathrm{GV}$ and becomes identical to the spectral indices of $\mathrm{He}, \mathrm{C}$, and $\mathrm{O}$ above $\sim 700 \mathrm{GV}$. Figure 2 shows the AMS nitrogen flux as a function of kinetic energy per nucleon $E_{K}$ together with the results of earlier measurements [4-7] and the prediction of a cosmic ray propagation model, GALPROP [10], which is based on data available before AMS. Data from earlier measurements have been extracted using Ref. [26]. For the AMS measurement $E_{K}=\left(\sqrt{Z^{2} \tilde{R}^{2}+M^{2}}-M\right) / A$ where $Z, M$, and $A$ are the nitrogen charge, mass, and atomic mass number, respectively. For comparison with other measurements the atomic mass number of 14.5 was used.

To examine the difference in the rigidity dependence of the nitrogen flux with respect to the fluxes of primary cosmic rays, the nitrogen to oxygen flux ratio $\mathrm{N} / \mathrm{O}$ was computed using the data in Table I of the Supplemental Material [16] and the data from Ref. [8] and is reported in Table II of the Supplemental Material [16] as a function of rigidity with its statistical and systematic errors. To provide a comparison with previous measurements, the $\mathrm{N} / \mathrm{O}$ ratio as a function of $E_{K}$ was computed using the procedure described in Ref. [21] and shown in Fig. 8(a) of the Supplemental Material [16] with total errors as a function of $E_{K}$ together with earlier measurements [4-7,27,28].

To examine the rigidity dependence of the nitrogen flux with respect to secondary cosmic rays, the nitrogen to boron flux ratio N/B was computed using data in Table I of the Supplemental Material [16] and data from Ref. [9] and is reported in Table III of the Supplemental Material [16] with its statistical and systematic errors as a function of rigidity. Figure 8(b) of the Supplemental Material [16] shows the AMS N/B ratio with total errors as a function of $E_{K}$ together with the results of earlier measurements [4-6].

To obtain the fractions of the primary $\Phi_{\mathrm{N}}^{P}$ and secondary $\Phi_{\mathrm{N}}^{S}$ components in the nitrogen flux $\Phi_{\mathrm{N}}=\Phi_{\mathrm{N}}^{P}+\Phi_{\mathrm{N}}^{S}$, a fit of $\Phi_{\mathrm{N}}$ to the weighted sum of a characteristic primary cosmic ray flux, namely, oxygen $\Phi_{\mathrm{O}}[8]$, and of a characteristic secondary cosmic ray flux, namely, boron $\Phi_{\mathrm{B}}$ [9], was performed over the entire rigidity range, as shown in Fig. 3(a). The fit yields $\Phi_{\mathrm{N}}^{P}=(0.090 \pm 0.002) \times \Phi_{\mathrm{O}}$ and $\Phi_{\mathrm{N}}^{S}=(0.62 \pm 0.02) \times \Phi_{\mathrm{B}} \quad$ with $\quad$ a $\quad \chi^{2} /$ d.o.f. $=51 / 64$. Figures 3(b) and 3(c) illustrate the result of this fit in the $\mathrm{N} / \mathrm{O}$ and N/B ratios, respectively. Figure 9 of the Supplemental Material [16] shows the contributions of the primary and secondary components in the nitrogen flux as functions of rigidity. As seen from Fig. 9 of the Supplemental Material [16], the contribution of the secondary component in the nitrogen flux drops from $70 \%$ at a few $\mathrm{GV}$ to below $30 \%$ above $1 \mathrm{TV}$. To verify the stability of the result, the fit was repeated in the rigidity range above $60 \mathrm{GV}$ yielding $\Phi_{\mathrm{N}}=(0.083 \pm 0.005) \times \Phi_{\mathrm{O}}+(0.66 \pm 0.04) \times \Phi_{\mathrm{B}}$ with a $\chi^{2} /$ d.o.f. $=18 / 25$, fully compatible with the results obtained with the fit over the entire rigidity range.

The observation that the nitrogen flux can be fit over a wide rigidity range as the simple linear combination of primary and secondary fluxes is a new and important result, which permits the determination of the N/O abundance ratio at the source without the need to consider the Galactic propagation of cosmic rays.

Finally, Fig. 4 shows the three distinctly different rigidity dependencies above $30 \mathrm{GV}$ of the primary $\mathrm{He}, \mathrm{C}$, and $\mathrm{O}$ cosmic ray fluxes, the secondary $\mathrm{Li}, \mathrm{Be}$, and $\mathrm{B}$ fluxes, and the $\mathrm{N}$ flux.

In conclusion, a precision measurement of the nitrogen flux in cosmic rays from $2.2 \mathrm{GV}$ to $3.3 \mathrm{TV}$ with detailed studies of the systematic errors has been presented. The flux deviates from a single power law. Its spectral index rapidly hardens at high rigidities starting from $\sim 100 \mathrm{GV}$ and becomes identical to the spectral indices of primary cosmic rays $\mathrm{He}, \mathrm{C}$, and $\mathrm{O}$ above $\sim 700 \mathrm{GV}$. Remarkably, the nitrogen flux is well described over the entire rigidity range by the sum of the primary flux $\Phi_{\mathrm{N}}^{P}$ equal to $9 \%$ of the oxygen flux [8] and the secondary flux $\Phi_{\mathrm{N}}^{S}$ equal to $62 \%$ of the boron flux [9]. This corresponds to a change of the contribution of the secondary component in the nitrogen flux from $70 \%$ at a few GV to below $30 \%$ above $1 \mathrm{TV}$.

We thank former NASA Administrator Daniel S. Goldin for his dedication to the legacy of the ISS as a scientific laboratory and his decision for NASA to fly AMS as a DOE payload. We also acknowledge the continuous support of the National Aeronautics and Space Administration (NASA) leadership including Charles Bolden and William H. Gerstenmaier and of the Johnson Space Center (JSC) and Marshall Space Flight Center (MSFC) flight control teams which has allowed AMS to operate optimally on the ISS for seven years. We are grateful for the support of Jim Siegrist and his staff of the DOE including resources from the National Energy Research Scientific Computing Center under Contract No. DE-AC02-05CH11231 and the Argonne Leadership Computing Facility under Contract No. DE-AC02-06CH11357. We also acknowledge the continuous support from MIT and its School of Science, Michael Sipser, Marc Kastner, Ernest Moniz, Richard Milner, and Boleslaw Wyslouch. Research supported by Sáo Paulo Research Foundation (FAPESP) Grants No. 2014/19149-7, No. 2015/50378-5, and No. 2016/10222-9, Brazil; CAS, NSFC, MOST, the provincial governments of Shandong, Jiangsu, Guangdong, and the China Scholarship Council, China; Action H2020 MSCA-IF-2015 under Grant No. 707543-MAtISSE, European Union; the Finnish Funding Agency for Innovation (Tekes) Grants No. 40361/ 01 and No. 40518/03 and the Academy of Finland Grant 
No. 258963, Finland; CNRS/IN2P3, CNES, Enigmass, and the ANR, France; Pascale Ehrenfreund, DLR under Grant No. $50 \mathrm{OO} 1403$ and JARA-HPC under Project No. JARA0052, Germany; INFN and ASI under ASIINFN Agreements No. 2013-002-R.0 and No. 2014-037R.0, Italy; the Consejo Nacional de Ciencia y Tecnología and UNAM, Mexico; FCT under Grant No. PTDC/FIS/122567/ 2010, Portugal; CIEMAT, IAC, CDTI, and SEIDI-MINECO under Grants No. ESP2015-71662-C2-(1-P/2-P), No. SEV2015-0548, No. MDM-2015-0509, and No. RyC-201314660, Spain; the Swiss National Science Foundation (SNSF), federal and cantonal authorities, Switzerland; Academia Sinica and the Ministry of Science and Technology (MOST) under Grants No. 103-2112-M-006018-MY3, No. 105-2112-M-001-003, and No. CDA-105M06, former Presidents of Academia Sinica Yuan-Tseh Lee and Chi-Huey Wong and former Ministers of MOST MawKuen Wu and Luo-Chuan Lee, Taiwan; the Turkish Atomic Energy Authority under Grants No. 2017TAEK(CERN) A5.H6.F2-15, Turkey; and NSF Grants No. 14255202 and No. 1551980, Wyle Laboratories Grant No. 2014/T72497, and NASA NESSF Grant No. HELIO15F-0005, USA. We gratefully acknowledge the strong support from CERN including Rolf-Dieter Heuer, Fabiola Gianotti, and the CERN IT department including Bernd Panzer-Steindel, and from the European Space Agency including JohannDietrich Wörner and Simonetta Di Pippo. We are grateful for important physics discussions with Fiorenza Donato, Jonathan Ellis, Jonathan Feng, Igor Moskalenko, Michael Salamon, Subir Sarkar, Joachim Trümper, Michael S. Turner, and Steven Weinberg.

${ }^{a}$ Also at ASI, I-00133 Roma, Italy.

${ }^{\mathrm{b}}$ Also at ASI Space Science Data Center (SSDC), I-00133 Roma, Italy.

${ }^{c}$ Also at Wuhan University, Wuhan 430072, China.

${ }^{\mathrm{d}}$ Also at Sun Yat-Sen University (SYSU), Guangzhou 510275, China.

eAlso at Nankai University, Tianjin 300071, China.

${ }^{\mathrm{f}}$ Also at Institute of Theoretial Physics, Chinese Academy of Sciences, Beijing 100190, China.

[1] H. A. Bethe, Phys. Rev. 55, 434 (1939).

[2] F. Vincenzo, F. Belfiore, R. Maiolino, F. Matteucci, and P. Ventura, Mon. Not. R. Astron. Soc. 458, 3466 (2016); C. Chiappini, D. Romano, and F. Matteucci, Mon. Not. R. Astron. Soc. 339, 63 (2003); R. B. C. Henry, M. G. Edmunds, and J. Koppen, Astrophys. J. 541, 660 (2000).

[3] I. A. Grenier, J. H. Black, and A. W. Strong, Annu. Rev. Astron. Astrophys. 53, 199 (2015); P. Blasi, Astron. Astrophys. Rev. 21, 70 (2013); A. W. Strong, I. V. Moskalenko, and V.S. Ptuskin, Annu. Rev. Nucl. Part. Sci. 57, 285 (2007); A. Castellina and F. Donato, Astrophys. Space Phys. Rev. 24, 1 (2005).

[4] M. Simon, H. Spiegelhauer, W. K. H. Schmidt, F. Siohan, J. F. Ormes, V. K. Balasubrahmanyan, and J. F. Arens, Astrophys. J. 239, 712 (1980); C. D. Orth, A. Buffington,
G. F. Smoot, and T. S. Mast, Astrophys. J. 226, 1147 (1978); E. Juliusson, Astrophys. J. 191, 331 (1974).

[5] J. J. Engelmann et al., Astron. Astrophys. 233, 96 (1990).

[6] S. Swordy, D. Mueller, P. Meyer, J. L'Heureux, and J. M. Grunsfeld, Astrophys. J. 349, 625 (1990).

[7] H. S. Ahn et al., Astrophys. J. 707, 593 (2009).

[8] M. Aguilar et al., Phys. Rev. Lett. 119, 251101 (2017).

[9] M. Aguilar et al., Phys. Rev. Lett. 120, 021101 (2018).

[10] We used Galprop webrun, A. E. Vladimirov, S. W. Digel, G. Jóhannesson, P. F. Michelson, I. V. Moskalenko, P. L. Nolan, E. Orlando, T. A. Porter, and A. W. Strong, Comput. Phys. Commun. 182, 1156 (2011); with parametrization from R. Trotta, G. Jóhannesson, I. V. Moskalenko, T. A. Porter, R. Ruiz de Austri, A. W. Strong, Astrophys. J. 729, 106 (2011).

[11] A. Putze, L. Derome, and D. Maurin, Astron. Astrophys. 516, A66 (2010)

[12] A. Kounine, Int. J. Mod. Phys. E 21, 1230005 (2012); S. Rosier-Lees, Proceedings of Astroparticle Physics TEVPA/ IDM, Amsterdam, 2014 (unpublished); S. Ting, Nucl. Phys. B, Proc. Suppl. 243-244, 12 (2013); S.-C. Lee, Proceedings of the 20th International Conference on Supersymmetry and Unification of Fundamental Interactions (SUSY 2012), Beijing, 2012 (unpublished); M. Aguilar, Proceedings of the XL International Meeting on Fundamental Physics, Centro de Ciencias de Benasque Pedro Pascual, 2012 (unpublished); S. Schael, Proceedings of the 10th Symposium on Sources and Detection of Dark Matter and Dark Energy in the Universe, Los Angeles, 2012 (unpublished); B. Bertucci, Proc. Sci., EPS-HEP, (2011) 67; M. Incagli, AIP Conf. Proc. 1223, 43 (2010); R. Battiston, Nucl. Instrum. Methods Phys. Res., Sect. A 588, 227 (2008).

[13] K. Lübelsmeyer et al., Nucl. Instrum. Methods Phys. Res., Sect. A 654, 639 (2011).

[14] B. Alpat et al., Nucl. Instrum. Methods Phys. Res., Sect. A 613, 207 (2010).

[15] V. Bindi et al., Nucl. Instrum. Methods Phys. Res., Sect. A 743, 22 (2014).

[16] See Supplemental Material at http://link.aps.org/ supplemental/10.1103/PhysRevLett.121.051103 for details of the AMS detector and data analysis; for the tabulated nitrogen flux, the nitrogen to oxygen and nitrogen to boron flux ratios as functions of rigidity; and for several figures regarding charge resolution and selection, survival probabilities, tracker residuals, other detailed systematic studies, the $\mathrm{N} / \mathrm{O}$ and $\mathrm{N} / \mathrm{B}$ ratios vs $E_{K}$ compared with earlier measurements, and the composition of $\Phi_{\mathrm{N}}$.

[17] G. Ambrosi, V. Choutko, C. Delgado, A. Oliva, Q. Yan, and Y. Li, Nucl. Instrum. Methods Phys. Res., Sect. A 869C, 29 (2017).

[18] M. Aguilar et al., Phys. Rev. Lett. 115, 211101 (2015).

[19] J. Allison et al., Nucl. Instrum. Methods Phys. Res., Sect. A 835, 186 (2016); S. Agostinelli et al., Nucl. Instrum. Methods Phys. Res., Sect. A 506, 250 (2003).

[20] M. Aguilar et al., Phys. Rev. Lett. 114, 171103 (2015).

[21] M. Aguilar et al., Phys. Rev. Lett. 117, 231102 (2016).

[22] J. Alcaraz et al., Phys. Lett. B 484, 10 (2000).

[23] C. C. Finlay et al., Geophys. J. Int. 183, 1216 (2010); E. Thébault et al., Earth Planet Space 67, 79 (2015).

[24] M. Aguilar et al. (AMS Collaboration), Measurement of the Flux of Light Nuclei in Primary Cosmic Rays with the 
Alpha Magnetic Spectrometer on the International Space Station (to be published).

[25] G. D. Lafferty and T. R. Wyatt, Nucl. Instrum. Methods Phys. Res., Sect. A 355, 541 (1995). We have used Eq. (6) with $\tilde{R} \equiv x_{l w}$.
[26] D. Maurin, F. Melot, and R. Taillet, Astron. Astrophys. 569, A32 (2014).

[27] H. S. Ahn et al., Astropart. Phys. 30, 133 (2008).

[28] A. D. Panov et al., Bull. Russian Acad. Sci. 73, 564 (2009). 